\title{
Emphasis of Periodicity in the Dynamic Behavior of Ionization Waves*)
}

\author{
Takao FUKUYAMA, Rina YAMAGUCHI and Hiroki KANZAKI \\ Faculty of Education, Nagasaki University, 1-14 Bunkyo-machi, Nagasaki 852-8521, Japan
}

(Received 28 November 2019 / Accepted 28 May 2020)

\begin{abstract}
The dynamic behavior of ionization waves in glow discharge plasma was examined, particularly with regard to periodicity. In a series of experiments, neon plasma was produced by the glow discharge between two electrodes after the glass tube was evacuated to high vacuum. Fluctuations in the light intensity were sampled with a line-scan camera and photodiodes as spatiotemporal signals for data analysis. The largest Lyapunov exponents were calculated from the time series of the experimental samples to quantitatively estimate the complexity of the system. The signal-to-noise ratio (SNR) became saturated as the intensity of the external force applied to the chaotic state was gradually increased because chaotic oscillations in the ionization wave synchronized to the external force. A periodic orbit in the chaos system was emphasized, and the oscillation became coherent as the state SNR reached its maximum value. When the periodicity was emphasized, the oscillation became coherent not only in time but also in space. Periodicity was also observed in system-induced feedback without an external force. Similar results were obtained with an external force and feedback; however, the former caused the chaos system to synchronize with the external force, while the latter yielded periodic oscillations from the chaos-chaos interaction.
\end{abstract}

(C) 2020 The Japan Society of Plasma Science and Nuclear Fusion Research

Keywords: glow discharge, ionization wave, chaos, periodicity, synchronization

DOI: $10.1585 /$ pfr. 15.2401049

\section{Introduction}

In the past decades, nonlinear phenomena of dissipative systems such as chaos, soliton, and fractals have gained considerable attention. Nonlinear phenomena have also attracted attention in plasma science because plasma is a typical medium that displays nonlinear properties [1-4]. This study focused on the dynamic behavior of ionization waves [5-7] in the positive column with glow discharge plasma. Various dynamic behaviors, such as chaos, have been observed in ionization waves as a function of discharge current. The spatiotemporal structures of ionization waves can be measured with detectors such as photodiodes and line-scan cameras. Therefore, ionization waves are a suitable medium for investigating the universal properties of nonlinearity.

Various types of oscillations can be observed in ionization waves. We focused on the stabilization of chaotic oscillation. The word stabilization is used in the sense that chaotic trajectory changes to periodic one. Chaos stabilization is an important topic for controlling plasma [8-10]. We examined the type of stabilization that causes an excitable system to respond to oscillation with the most coherence, i.e., the fundamental frequency of the external perturbation. Next, we experimentally consider the system's response to the strength of the applied force to con-

author'se-mail: fukuyama-takao@nagasaki-u.ac.jp

*) This article is based on the presentation at the 28th International Toki Conference on Plasma and Fusion Research (ITC28). trol the system when stabilization of chaos in plasma is accomplished. When perturbations, such as external force and feedback, are applied to the system to control it, a stronger force cannot always control the system. An extremely strong force may disrupt the stability of the system; however, even an imperceptible force may stabilize the system. Therefore, study on system's response (especially the emphasis on system's periodicity) depending on the strength of applied force is necessary as the next step.

In this paper, we report the dynamic behavior of ionization waves in plasma, particularly with regard to periodicity. Section 2 describes the setup and conditions for a series of experiments. Section 3 discusses the experimental results, especially with regard to periodicity depending on the application of an external sinusoidal force or feedback. Section 4 summarizes the findings of our study and concludes the paper.

\section{Experimental Setup}

Experiments were performed in a glass tube with a $2.0 \mathrm{~cm}$ diameter and $75 \mathrm{~cm}$ length. Ne gas was introduced into the glass tube at the pressure of approximately $478 \mathrm{~Pa}$ after the tube was evacuated to high vacuum. Ne plasma was produced by the glow discharge between two electrodes $60.0 \mathrm{~cm}$ apart when a DC electric field was applied. DC voltage is generated using a regulated DC power source (HV1.5-0.3, TAKASAGO). 
(a)

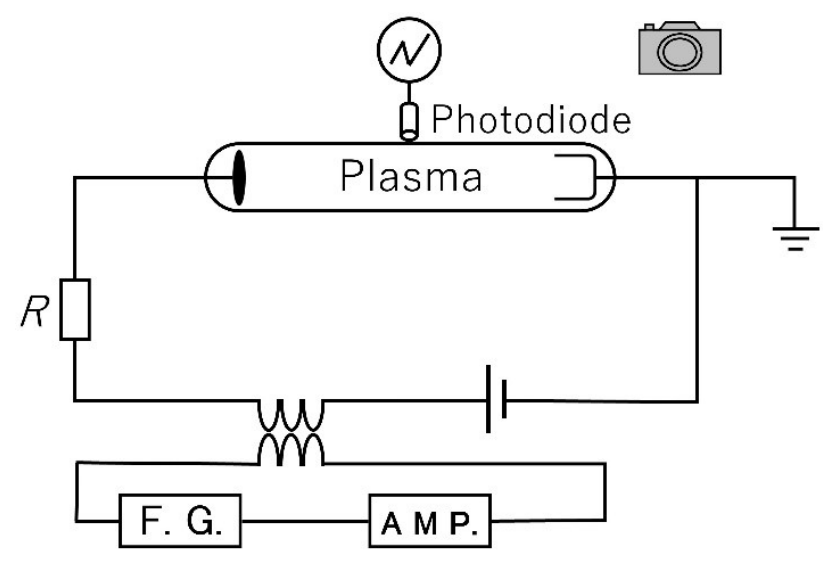

(b)



Fig. 1 Schematics of experimental configurations: (a) discharge tube applying an electric signal as an external force and (b) discharge tube influenced by feedback.

The typical ion and electron temperatures were $0.025 \mathrm{eV}$ and $10 \mathrm{eV}$, respectively. The intensity of the discharge current is a typical control parameter that governs the system. The system exhibited various nonlinear behaviors such as chaos and a periodic state. Light fluctuations along the central axis of the glass tube were sampled with photodiodes (S6775, HAMAMATSU), a linescan camera (TL-4096ACL, TAKENAKA), and a digital oscilloscope (GDS-1072A-U, GWINSTEK). External force is generated using a function generator (33220A, AGILENT). External force and feedback signal are amplified using a transformer (EF-4N, SHIMADZU) and an amplifier (4015, NF ELECTRONIC INSTRUMENTS). In most cases, the ionization waves showed the properties of a backward wave (i.e., the group and phase velocities were in opposite directions), although they sometimes showed the properties of a forward wave (i.e., the group and phase velocities were in the same direction).

Figure 1 shows schematics for the experimental configurations. Two types of setups were used: (a) a single discharge tube with an electric signal applied as an external force, and (b) a single discharge tube influenced by feedback. A photodiode was placed $30.0 \mathrm{~cm}$ away from the anode to observe the time series as fluctuations in the light intensity of Ne plasma. All Ne emissions were observed without an interference filter. Using a line-scan camera, the spatial and time series signals were obtained every $0.2 \mathrm{~mm}$ and $35.0 \mu \mathrm{s}$, respectively, and fluctuations in the light intensity were converted into 8-bit values. As shown in Fig. 1 (b), the feedback signal was sampled from light fluctuations that were $31.5 \mathrm{~cm}$ away from the anode. Two photodiodes were placed close together to observe the time series and sample the feedback signal at $30.0 \mathrm{~cm}$ and $31.5 \mathrm{~cm}$, respectively, from the anode.

\section{Results and Discussion}

The results corresponding to the experimental setup shown in Fig. 1 are presented here. Figure 2 shows the time series and power spectrum obtained from the (a) application of an external force and (b) influence of feedback. Figure 2 (a) shows the time series and power spectrum for the system with an applied external force; the upper trace shows the case before the application of an external force, and the lower trace shows the case during its application. The discharge current in the tube was fixed at $24.3 \mathrm{~mA}$, and an external sinusoidal force of $2.39 \mathrm{kHz}$ and $165.6 \mathrm{~V}$ was applied to the system. The frequency of the external force matched the fundamental frequency of the system. Figure 2 (b) shows the time series and power spectrum of the system with the feedback signal; the upper trace shows the case before the system is affected, and the lower trace shows the case when it is affected. The discharge current in the tube was fixed at $24.3 \mathrm{~mA}$, which was the same as that for the case shown in Fig. 2 (a). The voltage of the feedback signal, $118.0 \mathrm{~V}$, was measured according to the average peak-to-peak oscillation of the time series.

The time series and power spectra in Fig. 2 show that the system oscillation changed from chaotic to periodic. The fundamental frequency (approximately $2.39 \mathrm{kHz}$ ) produced a stronger power spectrum when the external force was applied. The system became coherent when responding to the oscillation of the fundamental frequency. In both systems, oscillations became coherent and periodic. However, the system in Fig. 2 (a) synchronized to the periodic external force, while the system in Fig. 2 (b) synchronized to the chaotic feedback oscillation.

Figure 3 shows the plots of signal-to-noise ratio (SNR) vs. the intensity of the (a) applied external force or (b) feedback (renormalized by the value of the discharge 
System

(a)
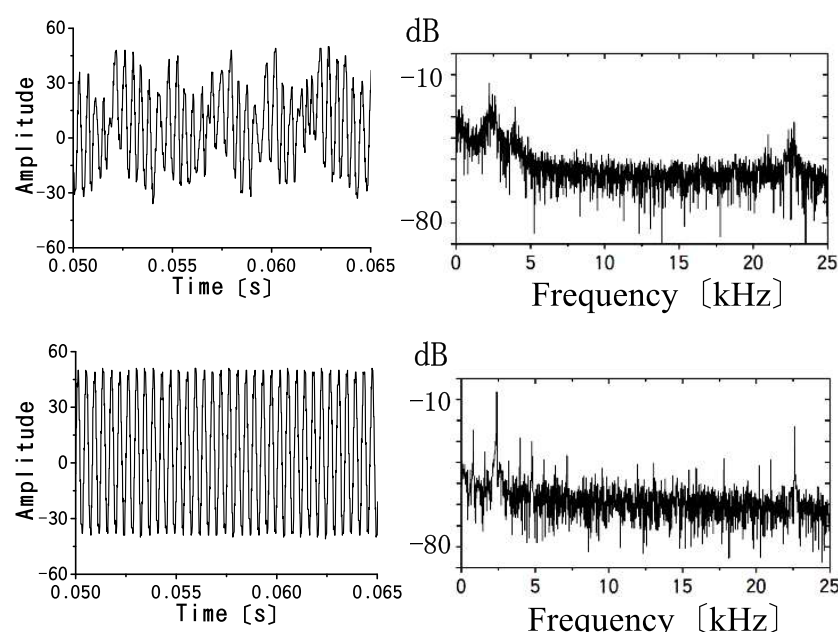

$\mathrm{dB}$

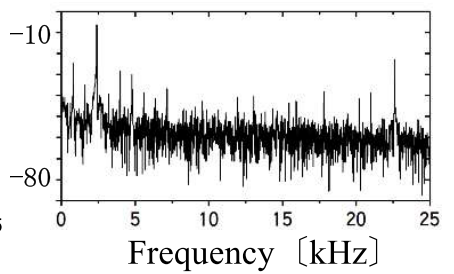

External force
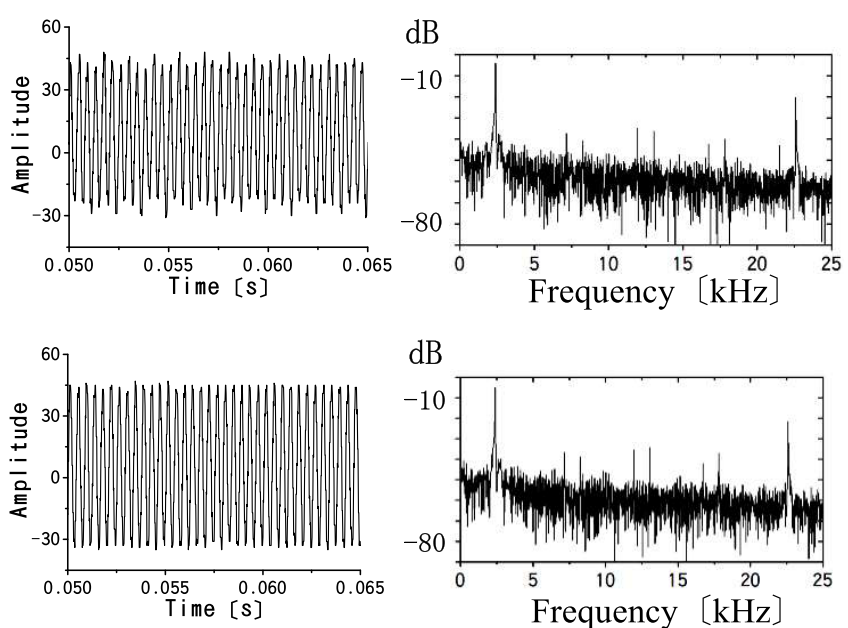

$\mathrm{dB}$

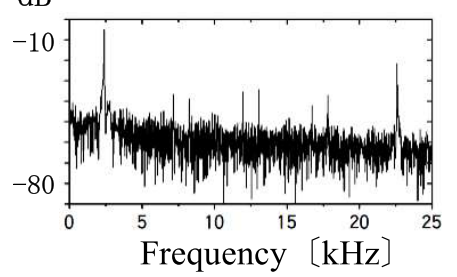

(b)

System

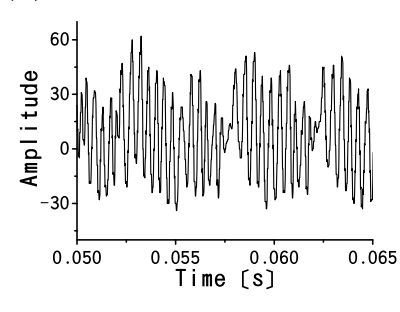

$\mathrm{dB}$
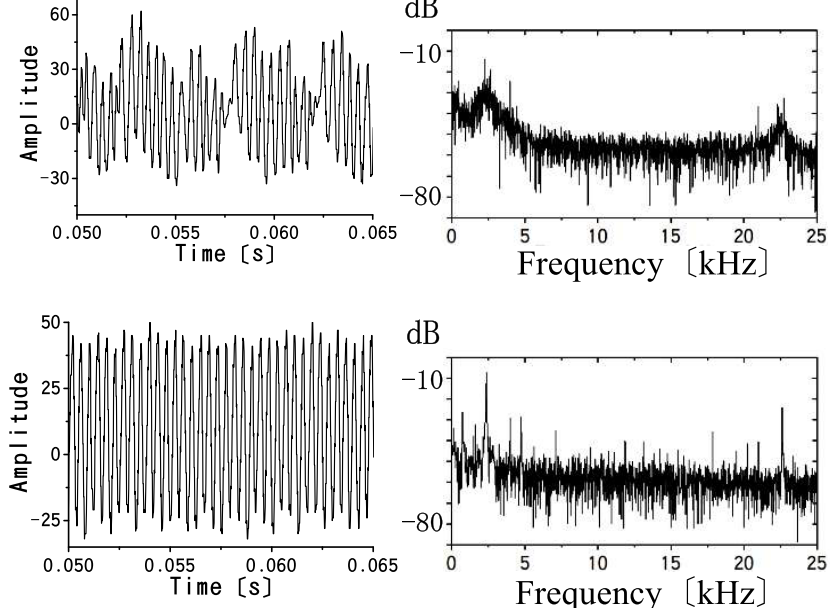
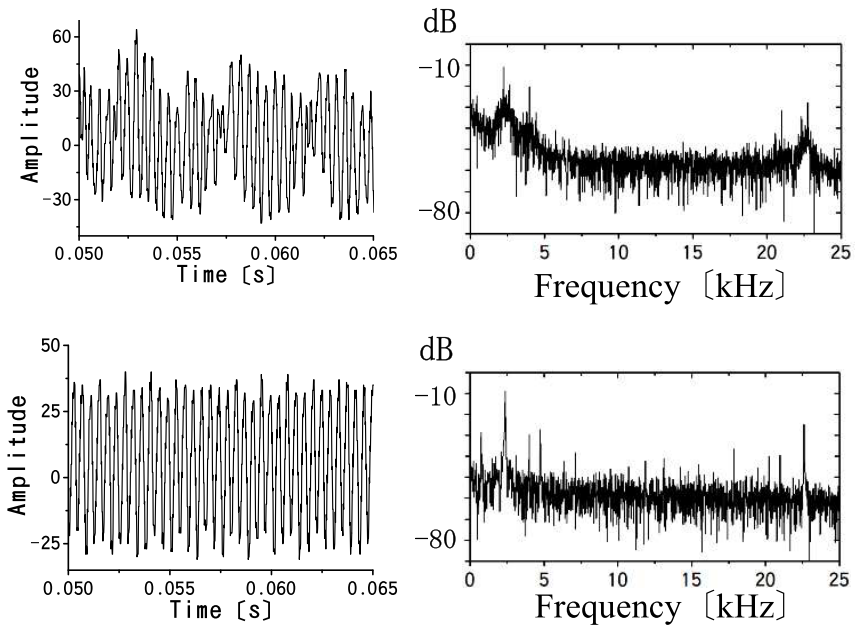

Fig. 2 Time series and power spectrum obtained from experiments: (a) application of an external force (2.39 kHz, 165.6 V) and (b) influence of feedback $(118.0 \mathrm{~V})$. The states before and during the process are shown by upper and lower traces, respectively. The discharge current in the tube was fixed at $24.3 \mathrm{~mA}$.

voltage, i.e., $605 \mathrm{~V})$. The SNR was calculated from the ratio of the power spectrum density of the peak frequency to the base noise level. The error bars represent the standard deviation, which was calculated from more than five measurements for each parameter.

With a gradual increase in the intensity of the applied force or feedback, the SNR became saturated at approximately the same value. When the intensity of the applied voltage was approximately 0.08 (61 V), the system changed from chaotic oscillation to periodic oscillation that took on coherence. An increase in the intensity of the external force or feedback considerably increased the perturbation in the ionization system, which increased the SNR. Above the threshold value, the oscillation became coherent, and the SNR became saturated.

To quantitatively examine the complexity of the system, the largest Lyapunov exponents were calculated for the time series sampled in the experiments using an algorithm developed by Wolf et al. [11]. For chaotic oscillations, the largest Lyapunov exponent had a positive value, which increased with the chaotic state. This value approached zero for a state with periodic oscillations. Figure 4 graphs the largest Lyapunov exponents vs. the intensity of the (a) applied external force and (b) feedback. The 
(a)

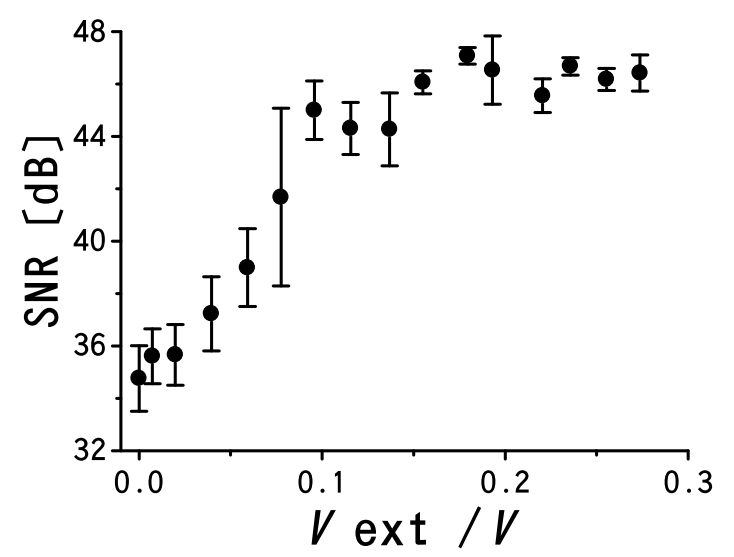

(b)

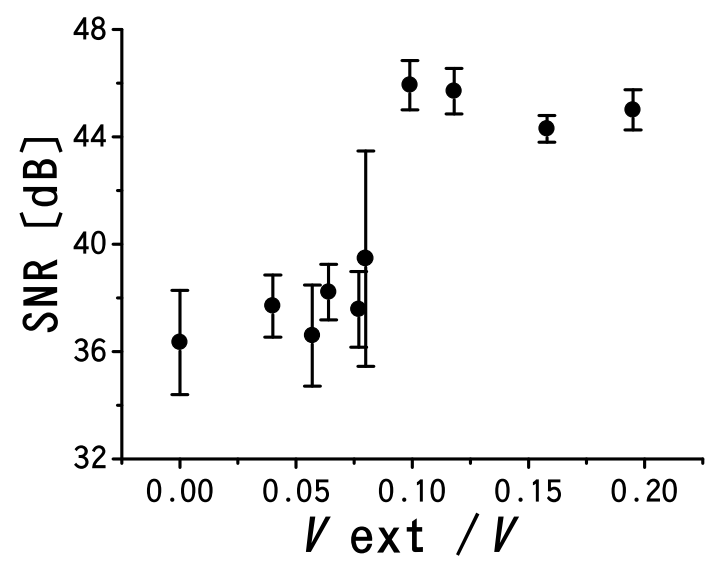

Fig. 3 Relationship between the intensity of the applied external force or feedback (renormalized by the value of the discharge voltage, i.e., $605 \mathrm{~V}$ ) and signal-to-noise ratio: (a) external force and (b) feedback. The error bars represent the standard deviation.

error bars represent the standard deviation, which was calculated from multiple measurements for each parameter.

The largest Lyapunov exponents decreased with an increase in the intensity of the applied voltage. Thus, the system stabilized when the applied voltage increased. By comparing Fig. 4 and Fig. 3, it was determined that the periodic orbit was emphasized when the applied external force or feedback was beyond the threshold.

Figure 5 shows typical changes in the experiments from spatiotemporal chaos to periodic structures: (a) the chaotic state without an external force or feedback and the discharge current fixed at $24.3 \mathrm{~mA}$, (b) the result from applying an external force, and (c) the influence of feedback. These results correspond to the states shown in Fig. 2. Figure 5 was generated from spatiotemporal data sampled using a line-scan camera, as previously mentioned.

Figure 5(a) indicates that the ionization system showed chaotic behavior in time and space. Bright lines in the spatiotemporal structure broke off, which indicated (a)

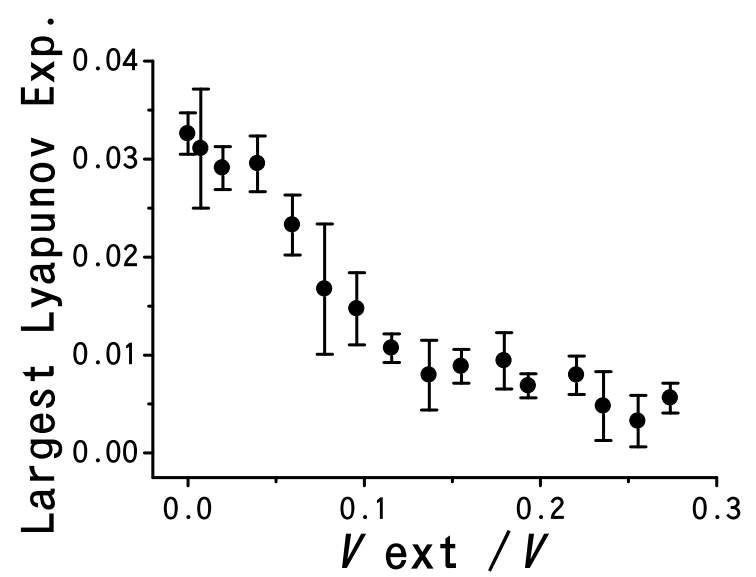

(b)

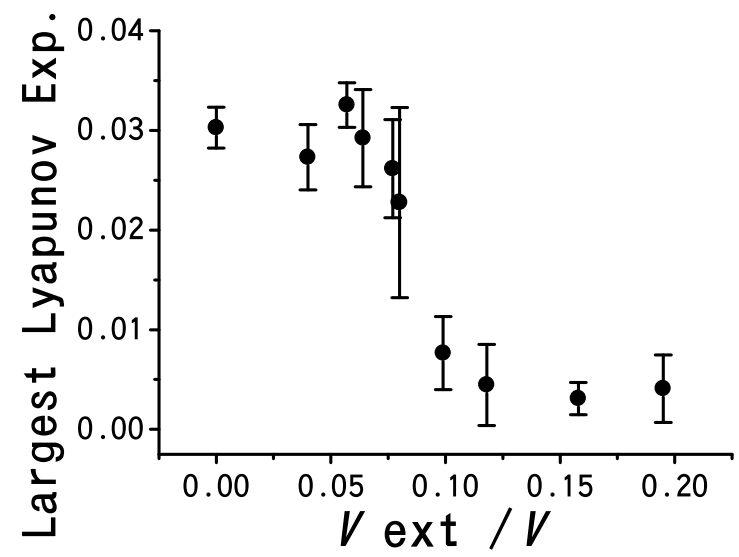

Fig. 4 Changes in the largest Lyapunov exponents: (a) with an external force applied and (b) the influence of feedback. The error bars represent the standard deviation.

that the system took on spatiotemporal chaos. Figures 5 (b) and (c) show that the ionization system exhibited periodicity and order in time and space. Bright lines clearly swept from the cathode (right) to anode (left) with regularity. When the temporal oscillation became periodic, the system became ordered not only in time but also in space.

\section{Conclusions}

A series of experiments was performed to examine the dynamic behavior of ionization waves, particularly with regard to periodicity.

(1) A gradual increase in the intensity of the external force applied to the chaotic state beyond a certain value caused the SNR, which was measured from the power spectrum, to become saturated.

(2) When the largest Lyapunov exponents were calculated, their values decreased with an increase in the intensity of the applied force. Thus, the system stabilized when the applied force was increased. 
(a)

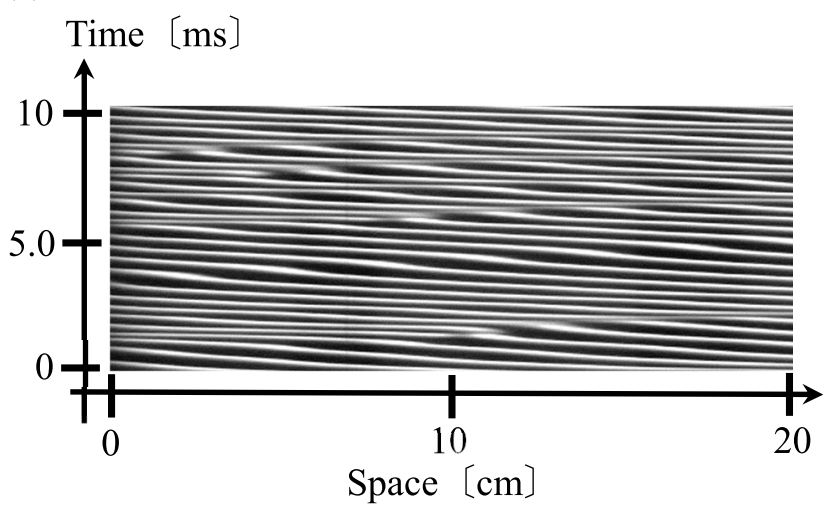

(b)

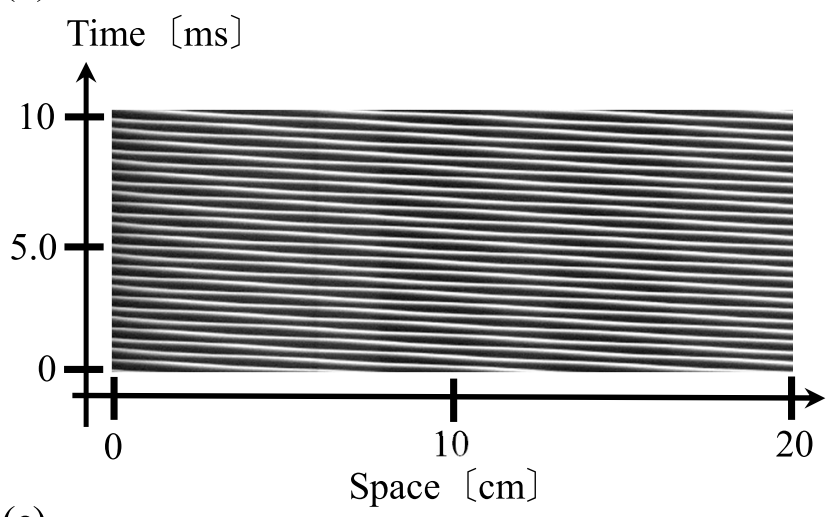

(c)

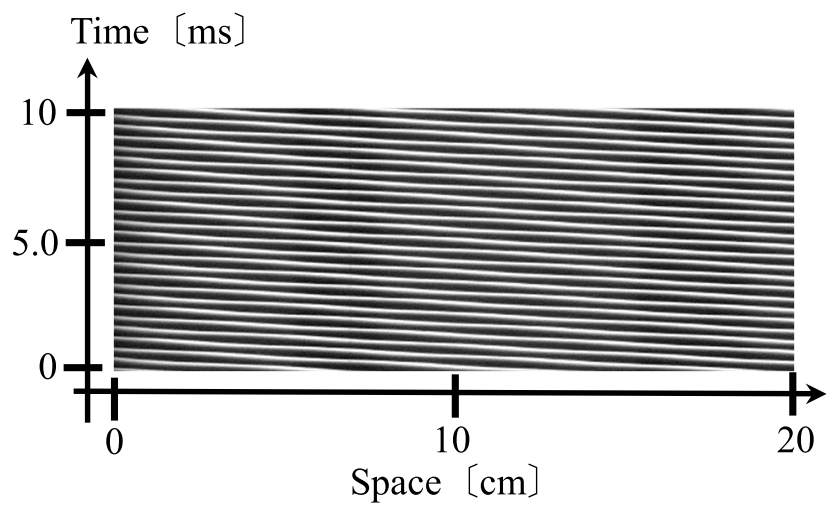

Fig. 5 Typical change from spatiotemporal chaos to periodic structures generated from spatiotemporal data sampled using a line-scan camera: (a) chaotic state with the discharge current fixed at $24.3 \mathrm{~mA}$, (b) application of an external force, and (c) influence of feedback.
(3) When the temporal oscillation in ionization waves became periodic when an external force was applied, the system became ordered not only in time but also in space.

(4) The periodicity described by points (1) - (3) was also observed in the system with induced feedback. Similar results were obtained for the two systems. However, the application of an external force caused the chaos system to synchronize with the external force, while the feedback yielded periodic oscillations caused by the chaos-chaos interaction.

This study is summarized as follows. The SNR and largest Lyapunov exponent saturate with an increase in the strength of applied force, i.e., external force and feedback. Therefore, an appropriate strength of applied force should be selected to bring periodicity to the chaotic system. A series of experiments were carried out by applying external force or feedback; however, the effect of application of noise is also an important aspect. As future work, study on the response of the system toward applied force and noise should be considered.

The authors express gratitude to the Reviewer for the fruitful discussions. This work is partly supported by JSPS KAKENHI Grant Number JP20K03895.

[1] W. Boswell, Plasma Phys. Control. Fusion 27, 405 (1985).

[2] Y. Cheung, S. Donovan and A.Y. Wong, Phys. Rev. Lett. 61, 1360 (1988).

[3] N. Ohno, M. Tanaka, A. Komori and Y. Kawai, J. Phys. Soc. Jpn. 58, 28 (1989).

[4] T. Fukuyama, K. Ota and H. Sakamoto, Phys. Plasmas 25, 092303 (2018).

[5] K. Ohe and S. Takeda, Contrib. Plasma Phys. 14, 55 (1974).

[6] Th. Pierre, G. Bonhomme and A. Atipo, Phys. Rev. Lett. 76, 2290 (1996).

[7] T. Fukuyama, K. Ishida and H. Kanzaki, Plasma Fusion Res. 14, 3401070 (2019).

[8] K. Pyragas, Phys. Lett. A 170, 421 (1992).

[9] T. Fukuyama, H. Shirahama and Y. Kawai, Phys. Plasmas 9, 4525 (2002).

[10] H. Tsuchiya, H. Shirahama and Y Kawai, Jpn. J. Appl. Phys. 46, 6074 (2007).

[11] A. Wolf, J.B. Swift, H.L. Swinney and J.A. Vastano, Physica D 16, 285 (1985). 